\title{
COMPLICACIONES TARDÍAS DE LA TERAPIA LÁSER COMO TRATAMIENTO DE LA TRANSFUSIÓN FETO-FETAL: CASO CLÍNICO
}

\author{
Mauro Parra C. ${ }^{1}$, Josefina Bascuñán A. ${ }^{a}$, Camila Valencia M. ${ }^{a}$, Gustavo Rencoret P. ${ }^{1}$, \\ Rafael Caballero T. ${ }^{1}$, Susana Quezada L. ${ }^{1}$ \\ 1 Unidad Medicina Materno-Fetal, Hospital Clínico Universidad de Chile.
}

a Alumnas de Medicina, Facultad de Medicina, Universidad de Chile.

\section{RESUMEN}

Presentamos la descripción del diagnóstico y manejo de una secuencia anemia-policitemia (SAP) que se presenta como complicación de una terapia láser exitosa en un embarazo gemelar monocorial cursando una transfusión feto-fetal (TFF) severa. Describimos la manifestación de esta complicación tardía de la terapia láser de la TFF severa y realizamos una revisión de la literatura internacional al respecto. A pesar del éxito de la introducción de la terapia láser en cuanto a la sobrevida y secuelas neonatales, recientemente se han descrito una serie de complicaciones de presentación tempranas o tardías. Entre las tardías, destacan la muerte de uno o ambos gemelos, recidiva de la TFF, y aparición de una SAP. Varios autores han descrito que la SAP sería secundaria a la presencia, o persistencia, de comunicaciones vasculares extremadamente pequeñas de flujo lento, las cuales llevan a una discordancia en los niveles de hemoglobina entre ambos gemelos, sin diferencias en sus volúmenes sanguíneos.

\section{PALABRAS CLAVE: Gemelar, transfusión feto-fetal, secuencia anemia-policitemia}

\section{SUMMARY}

We describe the diagnosis and management of twin anemia-polycythemia sequence (TAPS), which occurs as a late complication of successful laser therapy in twin monochorionic pregnancies developing severe twin to twin transfusion syndrome (TTTS). We offer a description of this late complication of laser therapy in this condition and a review of the related medical literature. Despite the successful introduction of laser therapy on the survival and neonatal sequelae, various early and late complications related to this procedure have been recently described. Among the late, stands out the death of one or both twins, recurrence of TTTS, and the appearance of TAPS. With regards TAPS, several authors have reported that it would be secondary to the presence, o persistence, of extremely small slow flow vascular communications, which lead to discrepancies in the hemoglobin levels between the twins, with no differences in blood volume.

KEY WORDS: Twins, twin to twin transfusion syndrome, twin anemia-polycythemia sequence 


\section{INTRODUCCIÓN}

Un tercio de los embarazos gemelares son monocigóticos, y de estos últimos dos tercios son monocoriales. Los embarazos gemelares monocoriales tienen un mayor riesgo perinatal que su contraparte bicorial, y esencialmente este mayor riesgo está dado por la presencia de complicaciones propias de la condición de monocorionicidad, tales como el síndrome de transfusión feto-fetal (STFF) y la restricción de crecimiento fetal selectiva (RCFs) (1).

EI STFF en sus distintos grados ocurre en alrededor de un $25 \%$ de los embarazos gemelares monocoriales, presentándose la condición severa en la mitad de ellos, o sea entre un 10 a $15 \%$ del total de los monocoriales (2). El diagnóstico se realiza básicamente, mediante la evaluación ecográfica de la discrepancia de líquido amniótico y vejiga en ambos sacos amnióticos y fetos, respectivamente (3). Quinteros y cols (4), diseñaron una clasificación que subdivide a los STFF en cuatro grupos de acuerdo a su pronóstico, la cual está basada en las características señaladas anteriormente y en la evaluación Doppler de la vasculatura fetal y placentaria. Por otro lado, la RCFs se define como la presencia de una discordancia mayor al $25 \%$ y/o uno de los gemelos con un peso bajo el percentil 10 , y su incidencia es también de un 15\% (5). En algunas oportunidades estas entidades pueden presentarse concomitantemente, y causar dificultades en el diagnóstico y manejo del caso. El pronóstico de estas condiciones se ha descrito adecuadamente en la literatura internacional y son dependientes del grado de predominio de una $u$ otra en el diagnóstico, y del grado de severidad de la condición específica (5).

EI STFF tiene una alta mortalidad, cercana al $90 \%$, y secuelas especialmente neurológicas en los sobrevivientes (6). Inicialmente la incorporación del amniodrenaje produjo una disminución de la mortalidad, pero manteniendo secuelas neonatales relativamente altas (7). Posteriormente, y aceptado en la actualidad como la terapia de elección en este tipo de casos, se introdujo la coagulación láser de las comunicaciones vasculares causantes del STFF. Esta terapia permite una sobrevida de por lo menos uno de los gemelos en el $76 \%$ de los casos, y una tasa de daño neurológico no superior al $2 \%$ (8). Sin embargo, a pesar del indudable cambio en el pronóstico de este tipo de gestaciones con la introducción de la terapia láser, esta técnica no está exenta de riesgos inmediatos y/o a largo plazo. Entre estas últimas, recientemente un grupo francés describió la aparición de complicaciones luego de los 7 días de un procedimiento considerado como exitoso, y las denominaron "tardías". Estas complicaciones son la secuencia anemia-policitemia (SAP), la reaparición de la TFF y la muerte de uno o ambos gemelos, las cuales tiene una incidencia cercana al $30 \%$ (3).

El objetivo de esta comunicación es presentar un caso de complicación tardía posterior a la coagulación láser exitosa en una paciente con STFF severa, asociada a RCFs.

\section{Caso clínico}

Paciente de 33 años, nulípara, con antecedentes de 2 abortos espontáneos, que se controló en la Unidad de Medicina Fetal del Hospital Clínico de la Universidad de Chile desde las 13 semanas de gestación, diagnosticándose un embarazo gemelar monocorial-biamniótico. En la evaluación ecográfica inicial se observó una discrepancia de translucidez nucal (2,6 vs. $1,9 \mathrm{~mm}$.) y longitud céfalo-nalgas (72 vs. $66 \mathrm{~mm}$ ) entre ambos gemelos, pero el ductus venoso y la regurgitación tricuspídea fueron normales. Se realizaron una serie de controles ecográficos desde las 16 semanas de gestación, sin signos evidentes de TFF y/o RCFs hasta las $20+5$ semanas donde presentó signos iniciales de TFF (Estadio I de Quinteros) y discrepancia de peso intergemelar de $30 \%$.

Posteriormente, en el control de las $22+5$ semanas, se planteó el diagnóstico de STFF estadio III de Quinteros, el cual se caracterizó por la presencia de una secuencia de polihidroamnios/anhidramnios, discrepancia en el tamaño de las vejigas (colapsada en el donante) y alteración del Doppler de arteria umbilical en el feto donante (flujo reverso en la arteria umbilical). Sin embargo, el ductus venoso en el receptor era normal. Además, y asociado al cuadro anterior, se estableció una discrepancia del peso intergemelar de $35 \%$. La anatomía fetal y longitud cervical se describieron como normales. Debido a estos hallazgos, se asesoró a la paciente en cuanto a las alternativas terapéuticas, proponiéndole como la mejor alternativa una terapia láser para coagular los vasos comunicantes de la superficie placentaria.

Se realizó una fetoscopia láser de 4 comunicaciones arterio-venosas desde el donante al receptor y 1 desde el receptor al donante en forma noselectiva. Al finalizar el procedimiento se procedió además a extraer 1,3 litros de líquido amniótico. La paciente evoluciona sin complicaciones, y el feto donante muestró una rápida recuperación de su hemodinamia, observándose aparición de vejiga, líquido amniótico y presencia de flujo diastólico en el Doppler de la arteria umbilical al día siguiente del 
procedimiento. El feto receptor, por otra parte, no muestró cambios significativos.

Los controles posteriores confirmaron la recuperación del desbalance hemodinámico, pero a los 14 días del procedimiento se observó un aumento de la velocidad máxima de la arteria cerebral media (VM-ACM) en el feto ex-receptor $(89,2 \mathrm{~cm} / \mathrm{seg}$; $>2,5 \mathrm{MoM}$ ), sugerente de anemia severa, y disminución de la VM-ACM en el ex-donante $(18,2 \mathrm{~cm} /$ seg; <0,7 MoM), sugerente de policitemia (Figura 1). Esta combinación de alteraciones en la hemodinamia de ambos fetos se consideró una SAP de aparición tardía.

Se discutió la complejidad del caso con la paciente y se plantearon dos alternativas de manejo: 1) repetir la coagulación láser y 2) transfusiones repetidas de glóbulos rojos en el feto anémico. Debido a que el segundo láser fue técnicamente dificultoso, se prosiguió el manejo con 4 transfusiones de glóbulos rojos en el gemelo anémico y una hemodilución en el policitémico hasta las $26+3$ semanas. La hemoglobina promedio del feto anémico fluctuó entre 2 y $4 \mathrm{~g} / \mathrm{dL}$, mientras que el feto policitémico tuvo una hemoglobina de $20 \mathrm{~g} / \mathrm{dL}$ (Figura 2).

Paralelo a lo anterior, y luego de la primera transfusión de glóbulos rojos, se detectó en ambos gemelos imágenes nodulares hiperecogénicas en el espesor del miocardio, lo cual fue sugerente de rabdomiomas miocárdicos.

Por último, debido a la persistencia de la anemia severa en el gemelo ex receptor y la imposibilidad de evaluar adecuadamente la condición hemodinámica del gemelo policitémico, se decidió realizar la interrupción del embarazo a las $26+4$ semanas de gestación por cesárea. Paradójicamente, el peso del gemelo ex-receptor fue menor al del feto policitémico, o ex-donante (710 vs. $730 \mathrm{~g}$ ), probablemente explicados por el exceso de glóbulos rojos transfundidos desde la circulación de su co-gemelo.

El manejo intensivo posnatal se presentó de manera muy compleja dada la labilidad hemodinámica de ambos gemelos, lo cual llevó al fallecimiento del gemelo anémico por falla cardiaca y multisistémica al sexto día de vida. La anatomía patológica practicada al gemelo fallecido, y la resonancia magnética en el sobreviviente, permitieron confirmar la presencia de múltiples rabdomiomas cardíacos y cerebrales (numerosas lesiones tuberosas corticales y astrocitoma subependimario), lo que configuró el diagnóstico de complejo de Esclerosis Tuberosa. Luego de 96 días de hospitalización se decidió el alta con oxigenoterapia (por displasia broncopulmonar) y control estricto de su evolución desde el punto de vista cardiaco y neurológico, por alto riesgo de convulsiones y de retraso mental.

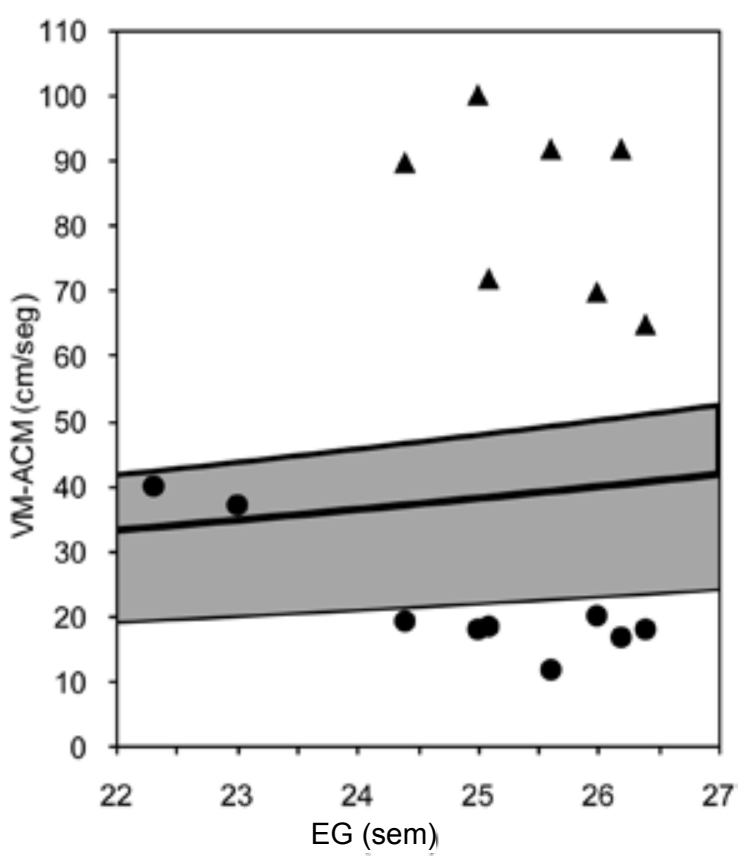

Figura 1. Variación de la velocidad máxima de la arteria cerebral media tanto en el gemelo afectado por anemia ( $\bullet$ ) como por policitemia $(\bullet)$ a distintas edades gestacionales.

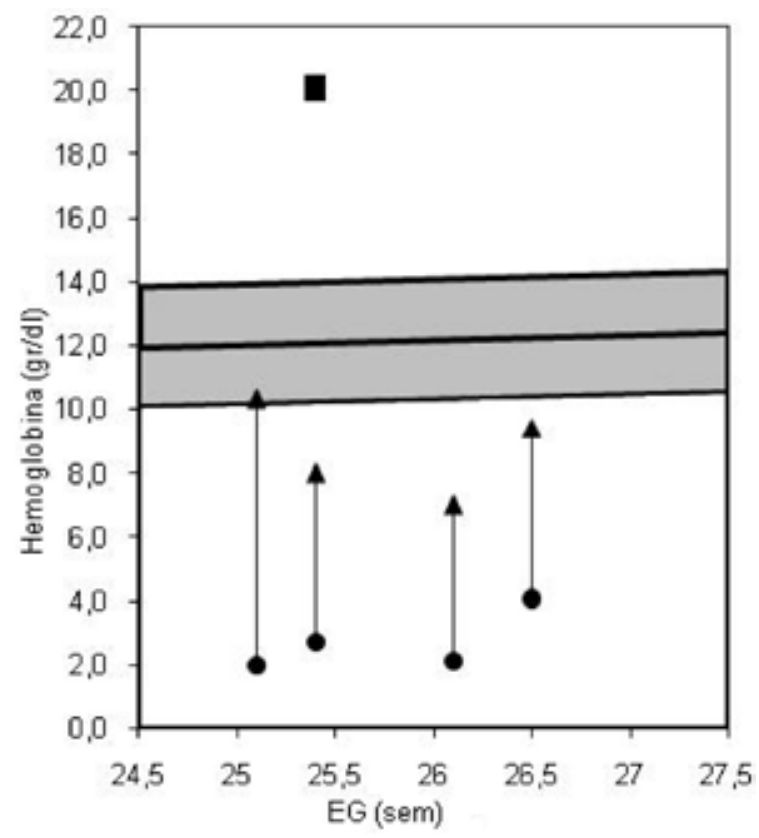

Figura 2. Expresión gráfica de la concentración de hemoglobina en el feto policitémico ( $\mathbf{0}$ ) y las variaciones pre $(\bullet)$ y pos $(\boldsymbol{\Delta})$ transfusionales obtenidas en el anémico. 
La evolución posterior del lactante ha sido satisfactoria, si bien persiste con oxigenoterapia, no ha presentado convulsiones ni complicaciones mayores y su desarrollo psicomotor ha sido adecuado. Aun así, el pronóstico sigue siendo incierto, debido principalmente a las múltiples lesiones cerebrales.

\section{DISCUSIÓN}

El caso presentado concuerda con las complicaciones tardías de la terapia láser en el STFF descritas en la literatura internacional (3). Según lo descrito en la literatura existen complicaciones tempranas o tardías de la terapia láser de la STFF, la cual alcanza al $20 \%$ de los casos (6). Por ello, se ha planteado que es importante realizar un seguimiento cercano de los casos tratados, no solo evaluando los parámetros biométricos, tamaño vesical y cantidad de líquido amniótico, sino que también adicionando la evaluación de la flujometría Doppler, con el objetivo de pesquisar precozmente complicaciones como una reaparición de TFF o SAP (3).

La SAP, complicación descrita en este caso clínico, definida como la presencia de anemia en uno de los fetos y policitemia en su co-gemelo, que se produciría por la presencia de una transfusión unidireccional desde el feto anémico hacia su co-gemelo.

El diagnóstico de SAP puede hacerse pre o posnatalmente. Los criterios diagnósticos prenatales consisten en la medición con flujometría Doppler de la velocidad máxima de la arteria cerebral media (VM-ACM) en ambos fetos. Los valores que configuran el diagnostico son, VM-ACM $>1,5$ $\mathrm{MoM}$ en el donante (sugerente de anemia severa) concomitante con VM-ACM $<0,8-1,0 \mathrm{MoM}$ en el receptor (sugerente de policitemia) (9). Según lo planteado, es importante realizar seguimiento con esta medición a todo embarazo monocorial, incluso aquellos que no presentan discordancia de volumen de líquido amniótico. Para hacer el diagnóstico posnatal, recientemente se ha sugerido considerar la diferencia de hemoglobina entre ambos gemelos mayor de $8 \mathrm{~g} / \mathrm{dL}$ sumado al menos a uno de los siguientes: relación de recuento reticulocitario entre gemelo donante/receptor > 1,7 y/o placenta con anastomosis vasculares menores a $1 \mathrm{~mm}$. Si bien prenatalmente, la SAP se presenta como un cuadro bastante menos frecuente que el STFF ( $2 \%$ vs. $10 \%$, respectivamente), con los datos posnatales, este cuadro alcanza el $4 \%$ de los embarazos monocoriales (10).

Se ha descrito que la SAP puede darse de manera espontánea (5-6\% de los embarazos monocoriales) o secundario a coagulación laser (13\%) (11). En los casos de complicaciones precoces o tardías de la terapia láser, ya sea STFF o SAP, se ha descrito la persistencia, o recanalización, de comunicaciones vasculares. Sin embargo, los factores que determinan que se produzca uno u otro cuadro, no han sido determinados con exactitud $(3,12)$.

Una eventual hipótesis ante la pesquisa de una SAP, luego de una terapia láser, podría ser a consecuencia de una coagulación no selectiva de los vasos comunicantes, lo cual lleva a una transfusión desde el feto receptor al donante durante el procedimiento láser. Sin embargo, si ésta fuera la causa de la anemia-policitemia observada en el caso descrito, la discrepancia hubiese aparecido precozmente en el seguimiento de la velocidad máxima de la ACM.

Otra hipótesis, y probablemente la que explicaría lo observado en este caso, es que la aparición tardía de SAP estaría mediada por una recanalización, o persistencia, de una escasa cantidad de comunicaciones arterio-venosas de calibres minúsculos. Estas comunicaciones facilitarían intercambios sanguíneos unidireccionales, lentos y persistentes en el tiempo, lo que asociado a la discrepancia de peso intergemelar, conducirían a la aparición progresiva de anemia en el ex receptor y policitemia en el ex donante $(3,6,13)$. Lopriore y cols $(14,15)$, estimaron que la velocidad del flujo en estas comunicaciones $\mathrm{A}-\mathrm{V}$ minúsculas debiese ser alrededor de $5-15 \mathrm{~mL} / 24$ horas.

Por otro lado, al igual que lo descrito en la literatura, en este caso no se observó alteración de los volúmenes de líquido amniótico, los cuales se habían normalizado luego de la terapia láser. Esta situación, que difiere del STFF, podría deberse nuevamente al flujo unidireccional extremadamente lento que persiste en estas minúsculas comunicaciones, lo cual facilitaría una compensación hemodinámica (16).

En cuanto al manejo de la SAP, se recomienda en primer lugar, realizar transfusión intrauterina en el feto anémico y extracción de sangre en el policitémico, cuando se requiera. Si esto fallase, podrían hacerse transfusiones seriadas, cirugía de las anastomosis recidivantes, feticidio selectivo del feto severamente afectado o interrupción del embarazo, dependiendo de la edad gestacional y el estado de los fetos $(3,12)$. La repetición de la fotocoagulación parece un manejo lógico, dado que la mayor proporción de casos se debe a comunicaciones vasculares, sin embargo, la detección de éstas en una segunda terapia láser suele ser técnicamente muy difícil. En el caso presentado, como se señaló anteriormente, se realizó una nueva coagulación láser, pero sin éxito. Posteriormente, el manejo continuó con transfusiones seriadas del feto anémico, hemodilución en el policitémico, e interrupción de la gestación cuando la situación hemodinámica de ambos 
gemelos se hizo crítica.

Aunque no hay asociación entre la presencia de los rabdomiomas cardíacos observados prenatalmente en este embarazo y la aparición de las complicaciones derivadas de la terapia láser, es necesario analizar brevemente esta malformación. La detección de rabdomiomas en el corazón de ambos fetos, mediante evaluación ecográfica, implica un $50 \%$ de probabilidades de presentar un complejo de Esclerosis Tuberosa, y un $50 \%$ de que solo se trate de un hallazgo aislado. Este caso cumplió los criterios, imagenológicos y anatomopatológicos, para ser catalogado como un complejo de Esclerosis Tuberosa. Esta enfermedad tiene una amplia gama de presentaciones clínicas, pudiéndose asociar a problemas neurológicos como epilepsia, retardo mental, alteraciones de comportamiento e incluso autismo, entre otros problemas, síntomas que pueden ir apareciendo a lo largo de los años (17).

\section{BIBLIOGRAFÍA}

1. Yamamoto M, Gratacós E, Ville Y. Gestación gemelar complicada (I): transfusión feto-fetal. En: Gratacós E, Gómez R, Nicholaides K, Romero R, Cabero L (eds). Medicina Fetal. Editorial Panamericana, 2007;695-702.

2. Quintero RA. Twin-twin transfusion syndrome. Clin Perinatol 2003;30:591-600.

3. Robyr R, Lewi L, Salomon LJ, Yamamoto M, Bernard JP, Deprest J, Ville Y. Prevalence and management of late fetal complications following successful selective laser coagulation of the chorionic plate anastomoses in twin-to-twin transfusion syndrome. Am J Obstet Gynecol 2006;194:796-803.

4. Quintero RA, Morales WJ, Allen MH, Bornick PW, Johnson PK, Kruger M. Staging of twin-twin transfusion syndrome. J Perinatol 1999;19:550-5.

5. Russell R, Quintero RA, Kontoupolus EV. Intrauterine growth restriction in monochorionic twins. Semin Fetal Neonatal Med 2007;12:438-49.

6. Yamamoto M, El Murr L, Robyr R, Leleu F, Takahashi $\mathrm{Y}$, Ville $\mathrm{Y}$. Incidence and impact of peri-operative complications in 175 fetoscopy-guided laser coagulations of chorionic plate anastomoses in fetofetal transfusion syndrome before 26 weeks of gestation. Am J Obstet Gynecol 2005;193:1110-6.

7. Quarello E, Molho M, Ville Y. Incidence, mechanisms and patterns of fetal cerebral lesions in twin-to-twin transfusion syndrome. J Matern Fetal Neonatal Med 2007;20:589-97.

8. Senat M, Deprest J, Boulvain M, Paupe A, Winer N, Ville $Y$. Endoscopic laser surgery versus serial amnioreduction for severe twin to twin transfusión syndrome. N Engl J Med 2004;351:136-44.

9. Slaghekke F, Kist WJ, Oepkes D, Pasman SA, Middeldorp JM, Klumper FJ, et al. Twin anemia-polycythemia sequence: diagnostic criteria, classification, perinatal management and outcome. Fetal Diagn Ther 2010;27:181-90.

10. Gucciardo L, Lewi L, Vaast P, Debska M, De Catte $\mathrm{L}$, Van Mieghem $\mathrm{T}$, et al. Twin anemia polycythemia sequence from a prenatal perspective. Prenat Diagn 2010;30:438-42.

11. Weingertner AS, Kohler A, Kohler M, Bouffet $N$, Hunsinger MC, Mager $C$, et al. Clinical and placental characteristics in four new cases of twin anemiapolycythemia sequence. Ultrasound Obstet Gynecol 2010;35:490-4.

12. Lopriore $\mathrm{E}$, Hecher $\mathrm{K}$, Vandenbussche FP, van den Wijngaard JP, Klumper FJ, Oepkes D. Fetoscopic laser treatment of twin to twin transfusion syndrome followed by severe twin anemia-polycythemia sequence with spontaneous resolution. Am J Obstet Gynecol 2008;198:e4-e7.

13. Lewi L, Jani J, Cannie M, Robyr R, Ville Y, Hecher K. Intertwin anastomoses in monochorionic placentas after fetoscopic laser coagulation for twin-to-twin transfusion syndrome: is there more than meets the eye? Am J Obstet Gynecol 2006;194:790-5.

14. Lopriore E, Middeldorp JM, Oepkes D, Kanhai HH, Walther FJ, Vandenbussche FP. Twin anemia-polycythemia sequence in two monochorionic twin pairs without oligopolyhydramnios sequence. Placenta 2007;28:47-51.

15. Lopriore E, van den Wijngaard JP, Middeldorp JM, Oepkes D, Walther FJ, van Genert MJ, et al. Assessment of fetofetal transfusion flow through placental arterio-venous anastomoses in a unique case of twin-to-twin transfusion syndrome. Placenta 2007;28:209-11.

16. Lopriore E, Deprest J, Slaghekke F, Oepkes D, Middeldorp JM, Vandenbussche FP, et al. Placental characteristics in monochorionic twins with and without twin anemia-polycythemia sequence. Obstet Gynecol 2008;112:753-8.

17. Roach ES, Gomez MR, Northrup H. Tuberous sclerosis complex consensus conference: revised clinical diagnostic criteria. J Child Neurol 1998;13;624-8. 